\title{
Neutron star-axion star collisions in the light of multimessenger astronomy
}

\author{
Tim Dietrich, ${ }^{1 \star}$ Francesca Day, ${ }^{2}$ Katy Clough, ${ }^{3}$ Michael Coughlin ${ }^{\oplus 4}$ and \\ Jens Niemeyer ${ }^{\oplus 3}$ \\ ${ }^{1}$ Nikhef, Science Park, NL-1098 XG Amsterdam, the Netherlands \\ ${ }^{2}$ DAMTP, Centre for Mathematical Sciences, Wilberforce Road, Cambridge CB3 OWA, UK \\ ${ }^{3}$ Institut für Astrophysik, Georg-August Universität, Friedrich-Hund-Platz 1, D-37077 Göttingen, Germany \\ ${ }^{4}$ Division of Physics, Math, and Astronomy, California Institute of Technology, Pasadena, CA 91125, USA
}

Accepted 2018 November 19. Received 2018 September 29; in original form 2018 August 20

\begin{abstract}
Axions are increasingly favoured as a candidate particle for the dark matter in galaxies, since they satisfy the observational requirements for cold dark matter and are theoretically well motivated. Fluctuations in the axion field give rise to stable localized overdensities known as axion stars, which, for the most massive, compact cases, are potential neutron star mimickers. In principle, there are no fundamental arguments against the multimessenger observations of GW170817/GRB170817A/AT2017gfo arising from the merger of a neutron star with a neutron star mimicker, rather than from a binary neutron star. To constrain this possibility and better understand the astrophysical signatures of a neutron star-axion star (NSAS) merger, we present in this work a detailed example case of an NSAS merger based on full 3D numerical relativity simulations, and give an overview of the many potential observables - ranging from gravitational waves to optical and near-infrared electromagnetic signals, radio flares, fast radio bursts, gamma ray bursts, and neutrino emission. We discuss the individual channels and estimate to what distances the current and future observatories might be able to detect such an NSAS merger. Such signals could constrain the unknown axion mass and its couplings to standard baryonic matter, thus enhancing our understanding of the dark matter sector of the Universe.
\end{abstract}

Key words: gravitational waves-hydrodynamics-methods: numerical-stars: neutrondark matter.

\section{INTRODUCTION}

The breakthrough discovery of GW170817 (Abbott et al. 2017a) with the combined detection of the gamma-ray burst GRB170817A (Abbott et al. 2017c) and the transient AT2017gfo (Abbott et al. 2017b) was the first coincident observation of gravitational waves (GWs) and electromagnetic (EM) waves from the same astrophysical source, and heralded a new era of multimessenger astronomy. While there is good evidence that GW170817, GRB170817A, and AT2017gfo were created by the coalescence and merger of two neutron stars (NSs) (Abbott et al. 2018a, b), it cannot yet be ruled out that the observed GW and EM signals came from the merger of an NS with an NS-mimicker. As shown in Cardoso et al. (2017) and Sennett et al. (2017) it is difficult to clearly distinguish NSs from exotic compact objects, e.g. boson stars (BSs), with second generation GW detectors.
BSs are stable solitonic solutions to the coupled Einstein-KleinGordon equations, which describe a massive scalar field in the presence of gravity. Axion stars (ASs) are a particular kind of BS - real scalar field oscillotons with additional self-interactions given by their non-trivial field potential $V(\phi)$. Axions, although still unobserved, are theoretically well motivated; they solve the strong CP problem of QCD (Peccei \& Quinn 1977), arise naturally in string theory compactifications (see e.g. Arvanitaki et al. 2010), and are promising candidates for dark matter (DM) [see Marsh (2016) for a comprehensive review]. ASs can form dynamically during the collapse of axion miniclusters in the early universe (Hogan \& Rees 1988; Kolb \& Tkachev 1993) in a process similar to galactic core formation in ultralight axion cosmologies (Schive, Chiueh \& Broadhurst 2014; Veltmaat, Niemeyer \& Schwabe 2018), by wave condensation (Levkov, Panin \& Tkachev 2018), or from non-standard primordial perturbations with enhanced small-scale power (Widdicombe et al. 2018). While these scenarios generally predict the substantial majority of axionic DM to remain unbound, or bound in ASs in the low-mass range, the high-mass tail of the AS mass

\footnotetext{
^E-mail: diettim@nikhef.nl
} 
fraction at low redshifts is widely unconstrained, motivating an exploration of observable signatures.

In this work, we build on the results of Clough, Dietrich \& Niemeyer (2018) in which a large number of different neutron star-axion star (NSAS) configurations were simulated. Although restricted to head-on collisions the simulations showed that for NSAS systems close to the threshold of $\mathrm{BH}$ formation a large fraction of the bosonic and baryonic material can be ejected from the system and that a significant release of GW energy occurs during the collision and the post-merger phase. Here we present a detailed case study of the observables from a particular NSAS merger based on the full 3D numerical relativity simulations. Using our simulation results, we also consider semi-analytically the potential conversion of axions to photons due to couplings to standard matter. Combined, these multimessenger signals would lead to a unique signature for NSAS mergers.

Note that to facilitate easier comparison with existing literature, we have used different unit systems for different multimessenger channels, i.e. we employ geometric units for describing the GW signal, cgs-units for the kilonova and radio observations, and Planck units for the discussion of observables caused by the conversion of axions to photons.

\section{CONFIGURATION AND MERGER DYNAMICS}

In this article, we consider the head-on collision of the NSAS system NSAS 007 of Clough et al. (2018). For completeness, we review the configuration's key properties here [see Clough et al. (2018) for a detailed discussion of the numerical methods and tests]. The initial configuration is based on the superposition of a single isolated NS and AS separated by $260 \mathrm{~km}$ along the $x$-axis of the numerical domain. We solve the constraint equations to obtain a solution consistent with general relativity (Clough et al. 2018; Dietrich, Ossokine \& Clough 2018a)

The NS, with a gravitational mass of $\sim 1.38 \mathrm{M}_{\odot}$ in isolation, employs a piecewise-polytropic fit of the SLy Equation of State (Douchin \& Haensel 2001; Read et al. 2009), which is in agreement with current constraints inferred from Abbott et al. (2017a). The AS employs the periodic cosine self-interaction potential $V(\phi)=$ $f_{\mathrm{a}}^{2} \mu^{2}\left(1-\cos \left(\phi / f_{\mathrm{a}}\right)\right)$ with the axion decay constant $f_{\mathrm{a}}$ (here set to $f_{\mathrm{a}}=0.5 \mathrm{M}_{\mathrm{pl}}$, for negligible self-interactions) and the axion length scale $\mu=m_{\mathrm{a}} c / \hbar$ (here set to $G M_{\odot} / c^{2}$, of order of the NS radius). The physical axion mass is denoted by $m_{\mathrm{a}}$ and for this system is of the order of $10^{-10} \mathrm{eV}$. We set the initial amplitude of the scalar field to $\phi_{\mathrm{c}} \approx 0.014$, which results in an AS mass of about $0.36 \mathrm{M}_{\odot} \cdot{ }^{1}$

In Fig. 1, we show the axion energy density (top part of each panel) and baryonic density (bottom part of each panel) during the simulation. At $t=9.3 \mathrm{~ms}$ (top panel), the AS is highly deformed due to the deep gravitational well of the NS, and bosonic matter extends over a large region of the computational domain. In contrast, the NSs shape is almost unchanged from its initial undisturbed state. At $t=$ $10.8 \mathrm{~ms}$ (middle panel), the AS and NS have merged and the highest axionic and baryonic energy density centres around the origin of the numerical domain. A fraction of the material becomes unbound and leaves the central region. At $t=17.6 \mathrm{~ms}$ (bottom panel) the remnant has a notable offset from the origin since the ejection of matter happens anisotropically such that the final object obtains a

${ }^{1}$ Widdicombe et al. (2018) find that for $f_{\mathrm{a}}=0.5 \mathrm{M}_{\mathrm{pl}}$ the formation of ASs in this mass range is one of the most favoured scenarios for their approximate model.
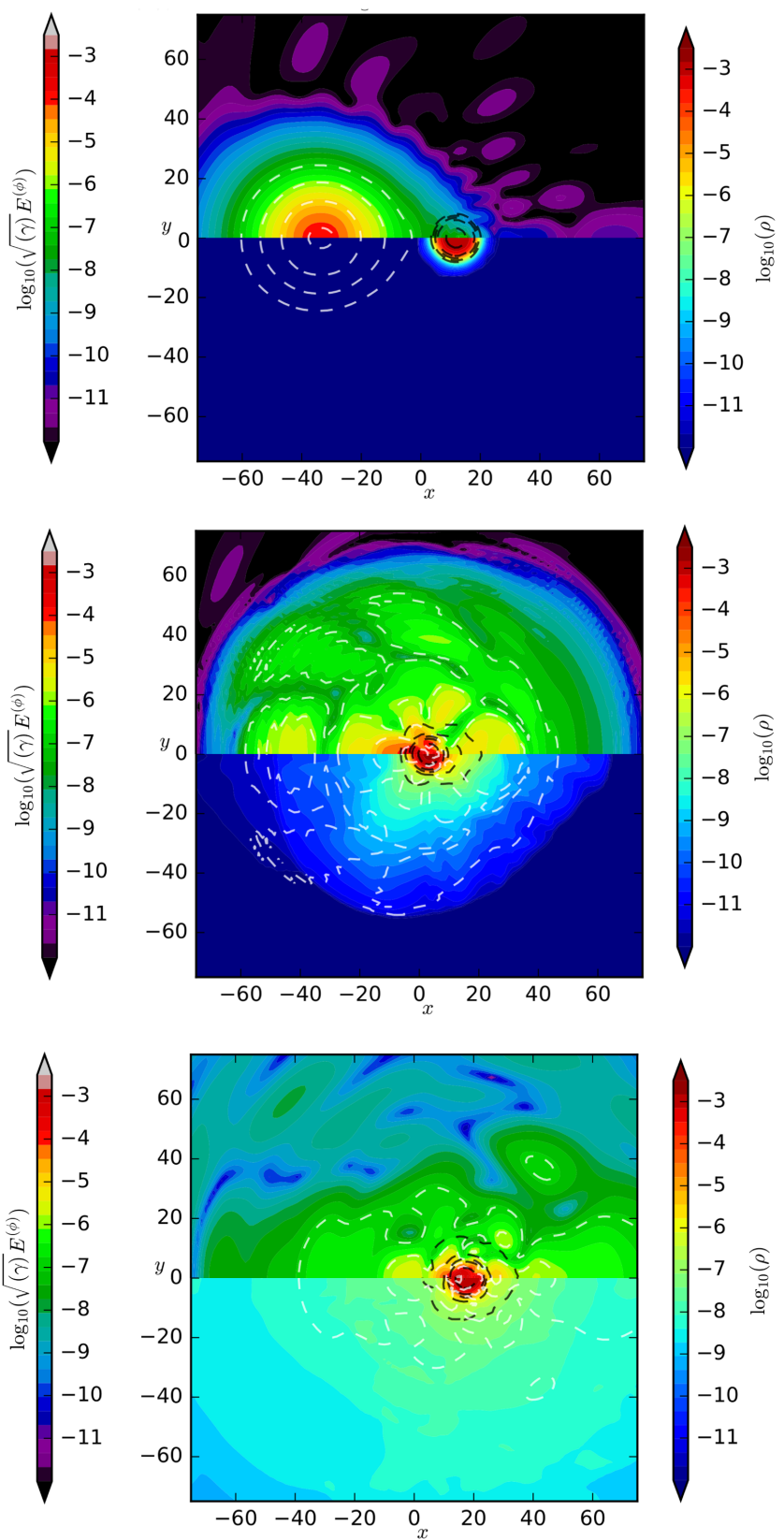

Figure 1. Energy density of the axionic matter (top part of each panel) and the baryonic density (bottom part of each panel) for the times $t=9.3,10.8$, $17.6 \mathrm{~ms}$. We also include contour densities lines corresponding to $10^{-7}$, $10^{-6}, 10^{-5}, 10^{-4}, 10^{-3}, 10^{-2}$ for the bosonic energy density (white dashed lines) and baryonic energy density (black dashed lines).

kick in the positive $x$-direction. The velocity of the remnant is of the order of $\sim 1000 \mathrm{~km} \mathrm{~s}^{-1}$ and thus comparable with the observation of high-velocity pulsars, e.g. Arzoumanian, Chernoffs \& Cordes (2002).

\section{THE MULTIMESSENGER PICTURE OF NSAS COLLISIONS}

\subsection{Gravitational wave emission}

In Fig. 2, we show for comparison the dominant (2,2)-mode of the GW signal for the NSAS configuration as well as for a BHAS and 


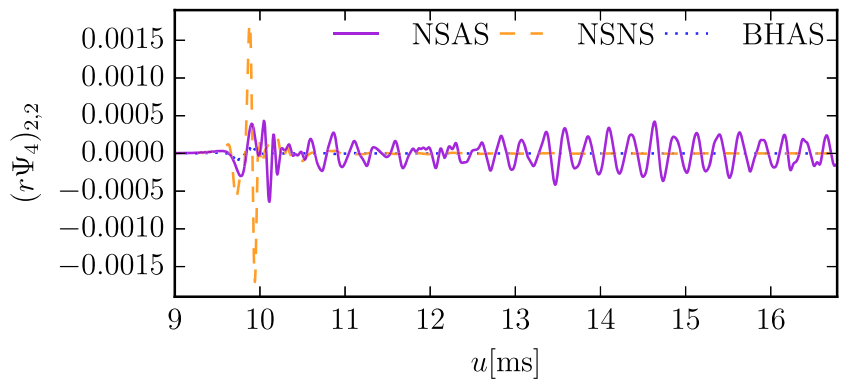

Figure 2. Dominant (2,2)-mode of the GW signal for the NSAS simulation as well as an NSNS and BHAS employing the same individual masses shown for comparison.

NSNS set-up using the same masses for the binary constituents. ${ }^{2} \mathrm{We}$ observe a clear ordering in the peak $\mathrm{GW}$ amplitude, which can be explained by the compactness of the individual objects. The smallest amplitude occurs for the BHAS merger, due to the large tidal distortion of the AS in the BH's gravitational field, with axionic matter falling into the $\mathrm{BH}$ before the actual merger. This effect is reduced for the NSAS configuration due to the smaller compactness of the NS compared to the BH. For a BNS merger, we find that tidal deformations are significantly smaller again and consequently, the merger amplitude is the largest. As is visible in the figure, the main differences in the GW emission with respect to a BNS system occur after the merger of the stars at frequencies for which current $\mathrm{GW}$ detectors are insensitive, see e.g. Bezares, Palenzuela \& Bona (2017). We find that GWs continue to be produced for the NSAS example case, such that the total energy emitted increases significantly over time. By the end of the simulation, the NSAS collision has released more energy in GWs than all the other cases, with a mass equivalent of the order $\mathcal{O}\left(10^{-3} \mathrm{M}_{\odot}\right)$. Assuming a burst search with current GW detectors our example head-on NSAS merger would only be observable up to $\sim 100 \mathrm{kpc}$ and for the Einstein Telescope (ET) up to $\sim 10 \mathrm{Mpc}$ (Abernathy et al. 2011).

Considering a potential quasi-circular NSAS merger, the emitted GW signal during the coalescence of an NSAS binary can be modelled in the same way as BNS systems and existing waveform models including tidal effects, e.g. Hinderer et al. (2016), Dietrich et al. (2018b), and Nagar et al. (2018) are already in place for their construction on quasi-circular orbits. ${ }^{3}$ For such a quasicircular merger, it would be possible to observe our example set-up up to $\sim 100 \mathrm{Mpc}$ for advanced LIGO at design sensitivity (Abbott et al. 2016) and possibly $\sim 1000 \mathrm{Mpc}$ for ET (Punturo et al. 2010).

\subsection{The kilonova from baryonic mass ejection}

As shown in Fig. 1(a) large fraction of bosonic and baryonic mass is ejected after the collision.

In particular the baryonic ejecta may give rise to an optical transient similar to AT2017gfo. To test this hypothesis, we measure the amount of unbound baryonic material and find that just $\sim 10 \mathrm{~ms}$ after the collision $\sim 10^{-2} \mathrm{M}_{\odot}$ of baryonic matter has been ejected.

\footnotetext{
${ }^{2}$ Note that due to the fact that the objects are placed along the $x$-axis, but the mode extraction is based on spin-weighted spherical harmonics defined along the $z$-axis, all $l=2$-modes are excited.

${ }^{3}$ We rely on Cardoso et al. (2017) and Sennett et al. (2017), who showed that it is possible to describe the deformation of exotic compact objects within an external gravitational field with the help of tidal deformability parameters, similarly to the description of NSs.
}

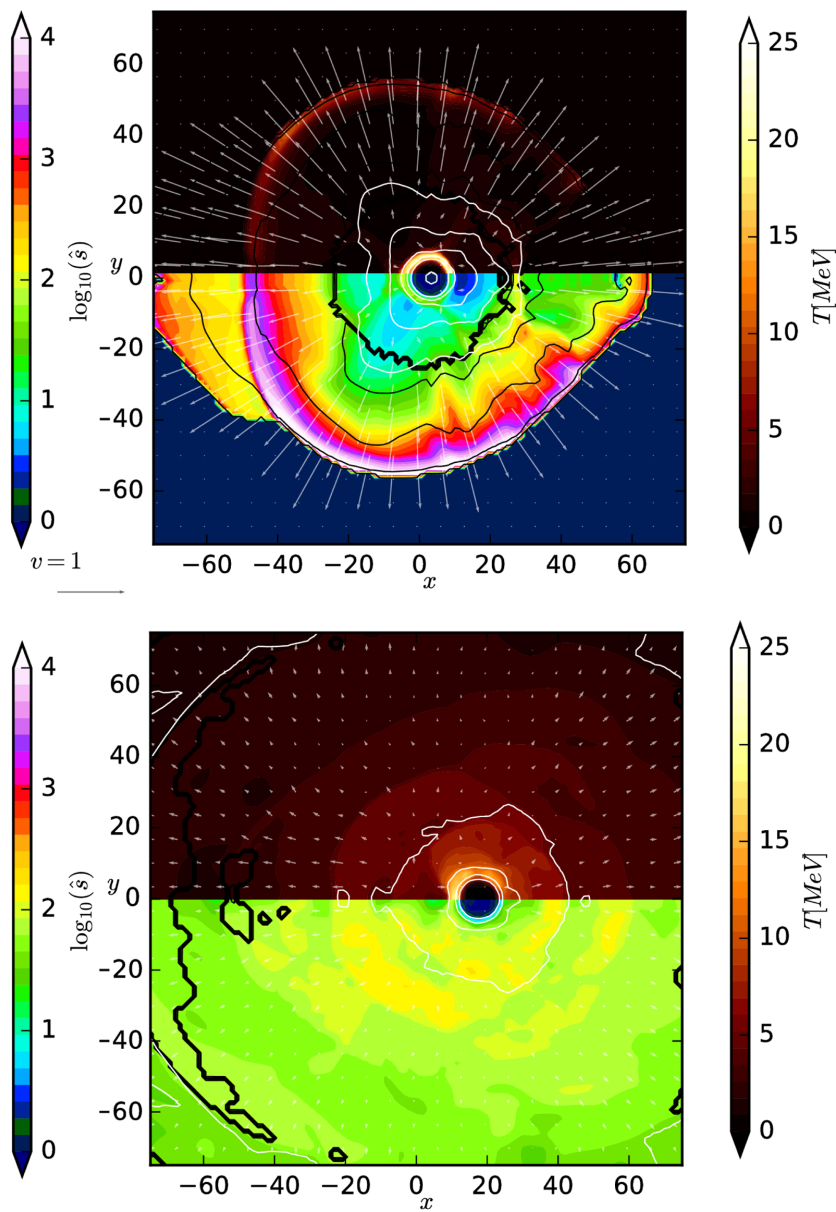

Figure 3. Temperature (top part of the panels) and entropy (bottom part of the panels) for the times $t=10.8 \mathrm{~ms}$ (top) and $t=17.6 \mathrm{~ms}$ (bottom), cf. Fig. 1. The white contour lines refer to the bound density, the black contours mark material that is unbound and ejected from the system.

At the end of the simulation the ejection rate is still of the order of $\sim 2 \times 10^{-3} \mathrm{M}_{\odot} \mathrm{ms}^{-1}$, i.e. ejecta will continue long after the simulated period. The main ejection mechanism for our configuration are shock waves formed during and after the merger of the two stars. In Fig. 3 (top panel) we show the temperature and entropy computed for the baryonic material. Both quantities can be estimated from our simulations, following Kyutoku (2013). Thermal effects are included in our simulations using an additional thermal pressure $p_{\text {th }}=\left(\Gamma_{\text {th }}-1\right) \rho \varepsilon_{\text {th }}$ where $\varepsilon_{\text {th }}$ denotes the thermal energy density and $\Gamma_{\text {th }}$ the thermal adiabatic exponent, which we set here to $\Gamma_{\text {th }}=1.75$ (cf. Bauswein, Janka \& Oechslin 2010). The entropy and temperature can then be estimated using

$\hat{s}=\frac{p}{p_{0}}=\frac{p_{0}+p_{\text {th }}}{p_{0}}$,

$\epsilon_{\mathrm{th}}=\frac{3 k T}{2 m_{u}}+\frac{11 a_{r} T^{4}}{4 \rho}$,

where $k$ is the Boltzmann constant, $m_{\mathrm{u}}$ the atomic mass unit, and $a_{\mathrm{r}}$ the radiation-density constant.

In Fig. 3 (top panel) we can clearly see the shock wave leaving the central region. Furthermore, we find that around the central (cold) region of the NS, temperatures of the order of $\sim 20 \mathrm{MeV}$ are reached. The temperature of the initial ejecta at the shock front 


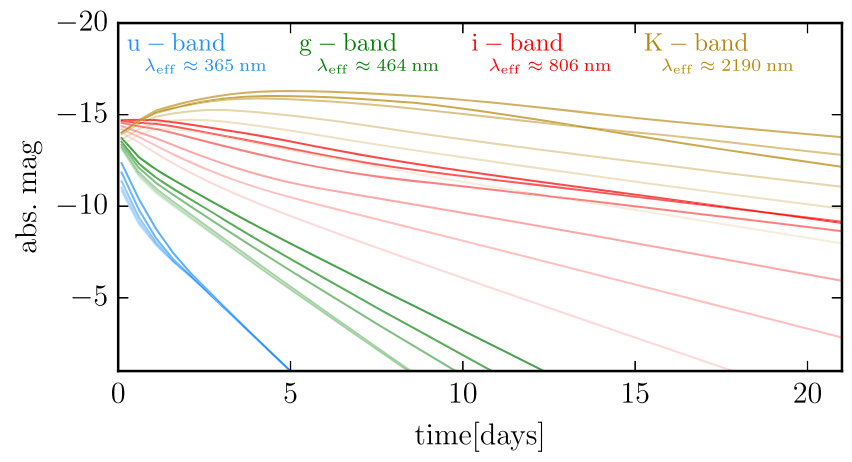

Figure 4. Absolute magnitudes in the $u$-, $g_{-}, i-, K$-band. The increasing opacity indicates an increasing total ejecta mass. We assume for the initial shock ejecta released shortly after merger an ejecta mass of $M_{\mathrm{ej}, 1}=$ $5 \times 10^{-3} \mathrm{M}_{\odot}$ and assume for the second ejecta component masses of $M_{\mathrm{ej}, 2}=5 \times 10^{-3} \mathrm{M}_{\odot}, 1 \times 10^{-2} \mathrm{M}_{\odot}, 2 \times 10^{-2} \mathrm{M}_{\odot}, 5 \times 10^{-2} \mathrm{M}_{\odot}$, $1 \times 10^{-1} \mathrm{M}_{\odot}, 2 \times 10^{-1} \mathrm{M}_{\odot}, 4 \times 10^{-1} \mathrm{M}_{\odot}$; see the text for more details.

is $\sim 10-15 \mathrm{MeV}$. Because of this high temperature, the first ejecta component released immediately after the merger (top panel of Fig. 3) will have a high electron and consequently low lanthanide fraction. The ejecta released later have a lower temperature, but are still shock driven.

To estimate the absolute magnitude of the transient based on the ejected baryonic material, we use the kilonova model of Kasen et al. (2017) and Coughlin et al. (2018a). The model of Kasen et al. (2017) employs a multidimensional Monte Carlo code to solve the multiwavelength radiation transport equation for a relativistically expanding medium. We interpolate between the existing Monte Carlo simulations using the Gaussian Process Regression techniques discussed in Coughlin et al. (2018a). For our estimates we assume the first ejecta component to have a mass of $M_{\mathrm{ej}, 1}=5 \times 10^{-3} \mathrm{M}_{\odot}$, an ejecta velocity of $v_{\mathrm{ej}, 1}=0.45 \mathrm{c}$, and a lanthanide fraction of $X=$ $10^{-3}$. For the remaining ejecta, we assume a velocity of $v_{\mathrm{ej}, 2}=$ $0.15 \mathrm{c}$ and a lanthanide fraction of $10^{-2}$. Since we do not know the total ejecta due to the finite length of our simulation, we vary the ejecta mass as $M_{\mathrm{ej}, 2}=5 \times 10^{-3} \mathrm{M}_{\odot}, 1 \times 10^{-2} \mathrm{M}_{\odot}, 2 \times 10^{-2} \mathrm{M}_{\odot}$, $5 \times 10^{-2} \mathrm{M}_{\odot}, 1 \times 10^{-1} \mathrm{M}_{\odot}, 2 \times 10^{-1} \mathrm{M}_{\odot}, 4 \times 10^{-1} \mathrm{M}_{\odot}$ and present different light-curve estimates in Fig. 4. The largest absolute magnitudes are obtained in the near-infrared, $\mathrm{cf}$. i- and $K$-band, while in the optical and ultraviolet the signal is fainter. Assuming that we use the Zwicky Transient Facility (Bellm 2014), which has a limiting magnitude of 20.4 in the $g$-band in its nominal survey mode (Coughlin et al. 2018b), then the transient would only be observable for the first few days after merger up to a distance of $\sim 40 \mathrm{Mpc}$. The Large Synoptic Survey Telescope (Ivezic et al. 2008) at its anticipated limiting magnitude of 24.4 in the $g$-band (Coughlin et al. 2018 b) could observe the NSAS merger transient up to $\sim 1.5 \mathrm{Gpc}$ (note that throughout we do not incorporate redshift corrections in the measured distances).

There are few wide-field surveys in the near-infrared, and so for comparison we instead highlight near-infrared imagers on largeaperture telescopes such as Gemini (Hodapp et al. 2003) with a limiting magnitude of around 22 in $K$-band in 30 s exposures. Due to the larger magnitude in the near-infrared, the transient would be observable for $\sim 2$ weeks up to a distance of $250 \mathrm{Mpc}$. Similarly, with the Thirty Meter Telescope's infrared imaging spectrograph (Wright et al. 2010), with a limiting magnitude of around 27 in $K$-band in 900 s exposures, the transient would be observable up to a distance of $\sim 2.5 \mathrm{Gpc}$.

\subsection{Radio flares from baryonic mass ejection}

The interaction between the interstellar medium and the mildly and sub-relativistic baryonic outflows will generate synchrotron radiation, so-called 'radio flares', (see e.g. Nakar \& Piran 2011). Radio flares triggered by the ejected material are observable for months or even years after the merger. For our NSAS configuration, the first ejecta component will lead to a peak in the radio band about $\sim 1 \mathrm{yr}$ after the merger with the radio fluence $\sim 28 \mathrm{Jy} /(D / \mathrm{Mpc})^{2}$. The more massive (but slower) ejecta component causes a peak in the radio band about $9 \mathrm{yr}$ after the merger with a fluence of $\sim 6.6 \mathrm{Jy} /(\mathrm{D} / \mathrm{Mpc})^{2}$. Comparing to the current capabilities of the Karl G. Jansky Very Large Array (VLA) with limiting fluxes of $\sim 10 \mu \mathrm{Jy}$, these objects are detectable out to $\sim 1 \mathrm{Gpc}$. With an approximately $100 \times$ higher sensitivity, the Square Kilometer Array (Murphy 2009) will detect such signals out to $\sim 10 \mathrm{Gpc}$.

\subsection{Neutrino emission because of shock heating}

Due to the fact that our numerical simulations do not include a full general relativistic radiation hydrodynamics scheme, we rely on simplified estimates to determine the neutrino luminosity. As shown in Fig. 3 the merger remnant reaches temperatures much larger than the electron-positron pair production threshold $(\sim 0.511 \mathrm{MeV})$. Consequently, positron captures $\left(n+e^{+} \rightarrow p+\bar{v}_{e}\right)$ lead to an increase of the electron fraction and the emission of electron-type antineutrinos.

In general there are two different mechanisms which could heat up the NS remnant during the NSAS collision. First, the shocks created during the merger process. Assuming that the neutrino emission happens within the hot region shown in Fig. 3 and that neutrinos with energies of $3 k T \sim 40 \mathrm{MeV}$ are emitted, we obtain a neutrino luminosity of the order of $10^{51} \mathrm{erg} \mathrm{s}^{-1}$. A second mechanism would be the conversion of axionic matter within the neutron star into thermal energy, which is discussed further below.

To estimate the amount of detectable neutrinos at detectors such as Super-Kamiokande (Fukuda et al. 2003) we use the relation

$R_{v}=\frac{21.1}{\mathrm{~ms}}\left[\frac{32 \mathrm{kT}}{M_{\mathrm{H}_{2} \mathrm{O}}}\right]\left[\frac{L_{\mathrm{nu}}}{10^{53} \mathrm{erg} \mathrm{s}^{-1}}\right]\left[\frac{E_{v}}{15 \mathrm{MeV}}\right]\left[\frac{10 \mathrm{kpc}}{D}\right]^{2}$

as given in Palenzuela et al. (2015). We find that the detection of neutrinos created due to shock heating during the collision of an NSAS merger will only be possible within our galaxy or in the near neighborhood of the Milky Way.

\subsection{Neutrino emission because of axion-photon heating}

While the multimessenger channels discussed so far also exist for a typical BNS merger, the presence of axions allows for additional signatures. In particular, the presence of an external magnetic field (as commonly observed for NSs) may lead to axion-photon interconversion (Raffelt \& Stodolsky 1988). While this process has been studied with respect to low-mass ASs that may be abundant in the Milky Way halo (Barranco, Monteverde \& Delepine 2013; Iwazaki 2015; Bai \& Hamada 2018), it has not been explored for an NSAS merger. 4

For the numerical relativity simulation, we assumed that the axion field was only coupled to baryonic matter via gravitational interaction, i.e. an interaction purely due to the mutual impact on the

\footnotetext{
${ }^{4}$ We note that if the axion in question is the QCD axion, its coupling to gluons may also lead to additional energy dispersion into the NS.
} 
metric. Based on the results of these simulations, we now discuss the effects of adding the typical coupling between the axion and electromagnetism. ${ }^{5}$ The accurate determination of these signals, discussed in this and the following section, would require full simulation of the relativistic axion-magnetohydrodynamics during the merger. We do not attempt to do this here. Instead, we seek to elucidate some of the possible effects, reviewing previously published approximations with the addition of some cautionary notes.

We first consider the effect of the AS in the bulk of the NS. The axion-photon interaction is described by the Lagrangian

$\mathcal{L} \supset-C \frac{\alpha}{f_{a}} \phi \mathbf{E} \cdot \mathbf{B}$,

with $\mathbf{E}, \mathbf{B}$, and $\alpha$ denoting the electric and magnetic fields and the fine structure constant, respectively. $C$ is a model-dependent constant of $\mathcal{O}(1)$. This Lagrangian leads to a modification of Maxwell's equations and Gauss's Law becomes

$\nabla \cdot \mathbf{E}=\rho-C \frac{\alpha}{f_{a}} \nabla \cdot(\phi \mathbf{B})$.

This leads to an axion-induced electric field in an electrically neutral medium $\mathbf{E}_{\phi}=-C \frac{\alpha}{f_{a}} \phi \mathbf{B}$, which creates a current $\mathbf{J}_{\phi}=\sigma \mathbf{E}_{\phi}$ in the NS (with the conductivity $\sigma$ ), as described in Barranco et al. (2013). This leads to a power dissipation into the NS

$P=\int \sigma E_{\phi}^{2} \mathrm{~d}^{3} x=\frac{C^{2} \alpha^{2} B^{2} \sigma}{f_{\mathrm{a}}^{2}} \int \phi(\mathbf{x})^{2} \mathrm{~d}^{3} x$.

For our example NSAS merger the AS envelopes the NS and thus the integral is taken over the entire NS. Note that for stable AS configurations, $\phi \propto f_{a}$, and therefore the power dissipated is independent of $f_{\mathrm{a}}$. With $\phi \sim 10^{-5} \mathrm{M}_{\mathrm{pl}}$, which is the typical field amplitude within the NS region at the end of the simulation, we can restrict our analysis to the leading order term in equation (4) and neglect the back reaction of the axion field on to the magnetic field since $\phi \ll f_{a}$. Taking a typical NS conductivity $\sigma \sim 10^{26} \mathrm{~s}^{-1}$ (Baiko \& Yakovlev 1995) we find

$$
\begin{aligned}
P(t=0)= & 10^{57} \mathrm{GeV} \mathrm{s}^{-1} \\
& \times C^{2}\left(\frac{B}{10^{8} \mathrm{~T}}\right)^{2}\left(\frac{\sigma}{10^{26} \mathrm{~s}^{-1}}\right)\left(\frac{R_{\mathrm{NS}}}{10 \mathrm{~km}}\right)^{3},
\end{aligned}
$$

where $R_{\mathrm{NS}}$ is the NS radius, and $t=0$ corresponds to the time of initial contact between the AS and the NS. In this case, the entire energy of the AS is released into the NS in $\sim 100 \mathrm{~ms}$.

However, we must also consider the dynamics of the growth of $\mathbf{E}_{\phi}$. Substituting Ohm's law into the axion-modified Maxwell equations, we obtain (Long \& Vachaspati 2015)

$\dot{\mathbf{E}}=\nabla \times \mathbf{B}-C \frac{\alpha}{f_{a}} \dot{\phi} \mathbf{B}-C \frac{\alpha}{f_{a}} \nabla \phi \times \mathbf{E}-\sigma \mathbf{E}-\sigma \mathbf{v} \times \mathbf{B}$,

where $\mathbf{v}$ is the fluid velocity. We therefore find that the growth of $\mathbf{E}_{\phi}$ could be almost entirely suppressed by the NS's high conductivity, in the absence of compensating contributions from other terms in equation (8), as discussed in Ahonen, Enqvist \& Raffelt (1996) for the case of axion DM in primordial magnetic fields. Furthermore, for a smaller $B$ or $C$ than assumed in equation (7), the energy dissipation would be slower. In this case, the AS may slowly heat the NS as they remain in contact after the merger. The energy released inside the bulk of the NS is unlikely to escape the NS directly as photons,

\footnotetext{
${ }^{5}$ Note that as the axion photon coupling is topological, its effects do not change in a curved spacetime.
}

but will increase the NS temperature, leading to increased neutrino emission.

In the case in which all the axions are converted, we obtain an increase of the NS's temperature of $\mathcal{O}\left(10^{2} \mathrm{MeV}\right)$ which leads to a neutrino luminosity $10^{52} \mathrm{erg} \mathrm{s}^{-1}$ of neutrinos with energy $\mathcal{O}(1 \mathrm{GeV})$. Using equation (3) we find that a detection of neutrinos up to $\sim 1 \mathrm{Mpc}$ is possible if the emission happens over a time of $1 \mathrm{~s}$. Using the sensitivity of the future Hyper-Kamiogrande neutrino observatory (Nakamura 2003), the neutrinos from axion conversion could be observable up to $\sim 20 \mathrm{Mpc}$.

\subsection{Electromagnetic bursts from axion-photon interconversion}

Unlike in the bulk, photons from axion to photon conversion in the magnetosphere of the NS may be observed directly. Furthermore, the conductivity of the magnetosphere may be significantly lower than that of the bulk (Li, Spitkovsky \& Tchekhovskoy 2012), and so the possible damping of $\mathbf{E}_{\phi}$ discussed above is avoided. We follow the approach of Iwazaki (2015) to estimate the size of this signal. As above, we have an axion-generated electric field $\mathbf{E}_{\phi}=\frac{-C \alpha \phi \mathbf{B}}{f_{a}} . \phi$ oscillates with a frequency corresponding to the axion mass $m_{\mathrm{a}}$, and so does $\mathbf{E}_{\phi}$. The electrons in the magnetosphere therefore oscillate parallel to $\mathbf{E}_{\phi}$, obeying the equation of motion $\dot{p}=e E$, with the electron's momentum $p$.

The electrons may become highly relativistic during these oscillations (Melrose \& Luo 2009; Reville \& Kirk 2010). Their maximum possible $\gamma$ factor is $\gamma_{\max }=\frac{p_{\max }}{m_{e}}=\frac{e E_{\phi}}{m_{a} m_{e}}=4.8 \times 10^{6}$. However, collisions between the electrons and other charged particles may reduce their maximum speed. If we assume the electron's speed returns to that determined by the local temperature after each collision, then relativistic speeds are obtained only at fewer than $10^{12}$ collisions per second. Furthermore, such collisions may result in the energy stored in the AS being dissipated as heat into the NS, rather than emitted as radiation, even in the magnetosphere.

The Larmor formula for radiated power $P=\frac{2 e^{4}}{3 m_{e}^{2}} E_{\phi}^{2}$ also applies for relativistic oscillations where acceleration is parallel to velocity. The emitted radiation is strongly beamed along the direction of the electron velocity, i.e. along the direction of $\mathbf{B}$. For dipole radiation for non-relativistic oscillating charged particles, the emitted radiation's frequency is the oscillation frequency. For axion species with masses below the mass considered here, this effect can be used to explain Fast Radio Bursts Iwazaki (2015). In the relativistic case, radiation is emitted at all frequencies up to a cut-off frequency $\omega_{c}=\gamma_{\max }^{2} m_{\mathrm{a}}$.

Therefore, rather than giving Fast Radio Bursts, higher mass NSAS mergers may lead to emission of EM radiation at a continuous range of frequencies.

We perform an order of magnitude estimate of the radiated power taking $\phi \sim 10^{-5} \mathrm{M}_{\mathrm{pl}}$, and assuming collisions within the magnetosphere are not significant. Using the Larmor formula, the power radiated from a single electron is $P_{1}=10^{-3} \mathrm{GeV} \mathrm{s}^{-1} \times C^{2}\left(\frac{B}{10^{8} \mathrm{~T}}\right)^{2}$. We take an electron density $n_{e}=10^{24} \mathrm{~cm}^{-3}$ (Chamel \& Haensel 2008) and a magnetosphere depth $d=1 \mathrm{~cm}$ (Ho \& Heinke 2009).

$$
\begin{aligned}
P= & 10^{33} \mathrm{GeV} \mathrm{s}^{-1} \\
& \times C^{2}\left(\frac{B}{10^{8} \mathrm{~T}}\right)^{2}\left(\frac{d}{1 \mathrm{~cm}}\right)\left(\frac{n_{e}}{10^{24} \mathrm{~cm}^{-3}}\right)\left(\frac{R_{\mathrm{NS}}}{10 \mathrm{~km}}\right)^{2} .
\end{aligned}
$$

In Iwazaki (2015), it is argued that the axion field oscillates coherently within its de Broglie wavelength, and therefore the total power output is enhanced by an extra factor of $N_{\mathrm{e}}$, the number of electrons in the de Broglie volume. This effect would lead to a 
factor of $10^{34}$ increase in the power output. However, the simulation is not sufficiently fine grained to test the coherence of the axion field, and furthermore the coherence of the oscillations could be destroyed by collisions within the magnetosphere. When the electrons obtain relativistic speeds, the majority of the emitted radiation is well above the plasma frequency of the magnetosphere, so we assume that a significant proportion will escape from the NS. The minimum duration of this EM burst will be $\mathcal{O}(100 \mathrm{~ms})$, the duration of the merger. For lower power outputs, the EM radiation may also be released over a longer period as the AS remains bound to the NS. The NSAS merger could therefore generate a distinctive fast burst of continuum radiation.

The observability of axion to photon interconversion in the magnetosphere depends on several factors. In particular, the NS magnetic field and the properties of collision in the magnetosphere determine the frequency of the emission, and therefore which telescopes could observe it. For example, let us consider the case in which the emission peaks at optical energies. Neglecting collisions, this corresponds to $B \sim 3 \times 10^{6} \mathrm{~T}$ and $\omega_{c}=2.1 \mathrm{eV}$. We optimistically assume a partial coherent enhancement of the signal, such that $P \propto N_{\mathrm{e}}^{1.5}$, and assume that all the resulting power dissipation escapes the magnetosphere.

In this case we have a radiated power $P=10^{47} \mathrm{GeV} \mathrm{s}^{-1}$. This power will be beamed along the direction of the magnetic field, which changes across the surface of the NS and with the NS's rotation. We therefore initially neglect the effect of beaming in this order of magnitude estimate. Assuming again the Zwicky Transient facility the burst of radiation lasting for about $100 \mathrm{~ms}$ might be seen up to a distance of $40 \mathrm{Mpc}$.

On the other hand, we could consider the most optimistic case in which the NS's magnetic dipole is pointing directly at the Earth, with most of the magnetic field lines parallel to the line of sight (LOS) throughout the NS's period. Taking $\gamma=10^{6}$, the GRB is then tightly beamed within $\gamma^{-1}$ along the LOS. In this somewhat unrealistic case, the burst calculation gives a distance of $90 \mathrm{Gpc}$, i.e. throughout the observable universe. We note that for a dipole model of the NS's magnetic field the beaming is very tight, within a cone of angle $\sim 10^{-6}$, i.e. it is likely that the GRB emission would be entirely beamed away from the LOS and be invisible to us. However, in the case where the NS magnetic field has a more complex structure, or the beam rotates on to the LOS during the merger due to the NS's rotation, it may be observable. For the Large Synoptic Survey Telescope we obtain maximum observable distances of $230 \mathrm{Mpc}$ and $580 \mathrm{Gpc}$ for the unbeamed and beamed scenario, respectively.

\section{SUMMARY AND OUTLOOK}

We have quantified the possibility of observing our example NSAS merger with different multimessenger channels, cf. Table 1.

Interestingly, we found that a number of observables usually connected with BNS or NSBH mergers are also present for NSAS mergers. This could lead to a misinterpretation of observable data, and so further studies identifying clear differences between BNSs and NSASs are required for a less ambiguous interpretation of future multimessenger observations. Consider for example GW170817 the optical/infrared/ultraviolet signature of AT2017gfo is broadly consistent with the kilonova signature from NSAS systems, and the signal sGRB170817 could be explained via formation of a BH and disc from an NSAS merger or from axion-photon conversion. We have also shown that NSAS mergers can release up to $M_{\mathrm{AS}}=10^{56}$ $\mathrm{GeV}$ in energy in the optical band for reasonable parameter values.
Table 1. A wanted poster: Multimessenger channels for a NSAS merger. The columns refer to the channel, the observable distance with current stateof-the-art techniques and the estimated observable distance with techniques anticipated for the near future.

\begin{tabular}{|c|c|c|}
\hline Channel & $D_{\text {today }}$ & $D_{\text {future }}$ \\
\hline $\mathrm{GW}_{\text {head-on }}$ & $\sim 0.1 \mathrm{Mpc}$ & $\sim 10 \mathrm{Mpc}$ \\
\hline $\mathrm{GW}_{\text {inspiral }}$ & $\sim 100 \mathrm{Mpc}$ & $\sim 1000 \mathrm{Mpc}$ \\
\hline Kilonova $_{\mathrm{u} \text {-band }}$ & $\sim 40 \mathrm{Mpc}$ & $\sim 1.5 \mathrm{Gpc}$ \\
\hline Kilonova $a_{K}$-band & $\sim 250 \mathrm{Mpc}$ & $\sim 2.5 \mathrm{Gpc}$ \\
\hline Radio flare & $\sim 1 \mathrm{Gpc}$ & $\sim 10 \mathrm{Gpc}$ \\
\hline Neutrino $_{\text {shock }}$ & $\sim 0.1 \mathrm{Mpc}$ & $\sim 2 \mathrm{Mpc}$ \\
\hline Neutrino $_{\text {axion-heating }}$ & $\sim 1 \mathrm{Mpc}$ & $\sim 20 \mathrm{Mpc}$ \\
\hline EM burst $\mathrm{t}_{\text {axions }- \text { photon }}^{\text {unbeamed }}$ & $\sim 40 \mathrm{Mpc}$ & $\sim 230 \mathrm{Mpc}$ \\
\hline EM burst $t_{\text {axions-phed }}^{\text {beamoton }}$ & $\sim 90 \mathrm{Gpc}$ & $\sim 580 \mathrm{Gpc}$ \\
\hline
\end{tabular}

Therefore, for some choices of the NS conductivity and magnetic field strength, a NSAS merger could give rise to unusual transients such as AT2018cow (Prentice et al. 2018), in which an unexplained optical luminosity of $\sim 44 \mathrm{ergs} \mathrm{s}^{-1}$ was observed.

The confirmed detection of an NSAS merger would be a significant discovery, simultaneously confirming the axion as a DM component, and constraining its mass, decay constant, and couplings to standard model particles. It would thus provide a lead in our understanding of the nature of dark matter within the Universe.

\section{ACKNOWLEDGEMENTS}

TD acknowledges support by the European Unions Horizon 2020 research and innovation program under grant agreement No 749145, BNSmergers. Computations have been performed on the supercomputer SuperMUC at the LRZ (Munich) under the project number pr48pu, and the compute cluster Minerva of the Max-Planck Institute for Gravitational Physics. This work has been partially supported by STFC consolidated grant ST/P000681/1. We thank David J E Marsh and MC David Marsh for useful discussions. Furthermore, TD and KC want to thank S.Khan for helpful comments on our simulations of ASs.

\section{REFERENCES}

Abbott B. P. et al., 2016, Phys. Rev., 93, 112004

Abbott B. P. et al., 2017a, Phys. Rev. Lett., 119, 161101

Abbott B. P. et al., 2017b, ApJ., 848, L12

Abbott B. P. et al., 2017c, ApJ., 848, L13

Abbott B. P. et al., 2018a, preprint(arXiv:1805.11579)

Abbott B. P. et al., 2018b, Phys. Rev. Lett., 121, 161101

Abernathy M. et al., 2011, Einstein gravitational wave Telescope: Conceptual Design Study, Avaiable at: ET-0106C-10

Ahonen J., Enqvist K., Raffelt G., 1996, Phys. Lett., 366, 224

Arvanitaki A., Dimopoulos S., Dubovsky S., Kaloper N., March-Russell J., 2010, Phys. Rev., 81, 123530

Arzoumanian Z., Chernoffs D. F., Cordes J. M., 2002, ApJ., 568, 289

Bai Y., Hamada Y., 2018, Phys. Lett., 781, 187

Baiko D. A., Yakovlev D. G., 1995, Astron. Lett., 21, 709

Barranco J., Monteverde A. C., Delepine D., 2013, Phys. Rev., 87, 103011

Bauswein A., Janka H. T., Oechslin R., 2010, Phys. Rev., 82, 084043

Bellm E., 2014, in Wozniak P. R., Graham M. J., Mahabal A. A., Seaman R., eds, The Third Hot-wiring the Transient Universe Workshop, p. 27, www.slac.stanford.edu/econf/C131113.1/proceedings.html

Bezares M., Palenzuela C., Bona C., 2017, Phys. Rev., 95, 124005

Cardoso V., Franzin E., Maselli A., Pani P., Raposo G., 2017, Phys. Rev., 95, 084014

Chamel N., Haensel P., 2008, Living Rev. Rel., 11, 10 
Clough K., Dietrich T., Niemeyer J. C., 2018, Phys. Rev., 98, 83020

Coughlin M. W. et al., 2018a, MNRAS, 480, 3871

Coughlin M. W. et al., 2018b, MNRAS, 478, 692

Dietrich T. et al., 2018b, preprint(arXiv:1804.02235)

Dietrich T., Ossokine S., Clough K., 2018a, preprint (arXiv:1807.06959)

Douchin F., Haensel P., 2001, A\&A, 380, 151

Fukuda Y. et al., 2003, Nucl. Inst. Methods Phys. Res., 501, 418

Hinderer T. et al., 2016, Phys. Rev. Lett., 116, 181101

Hodapp K. W. et al., 2003, PASP, 115, 1388

Hogan C. J., Rees M. J., 1988, Phys. Lett., 205, 228

Ho W. C. G., Heinke C. O., 2009, Nature, 462, 71

Ivezic Z., Tyson J. A., Allsman R., Andrew J., Angel R., 2008, SLAC-PUB16076

Iwazaki A., 2015, Phys. Rev., 91, 023008

Kasen D., Metzger B., Barnes J., Quataert E., Ramirez-Ruiz E., 2017, Nature, 551, 80

Kolb E. W., Tkachev I. I., 1993, Phys. Rev. Lett., 71, 3051

Kyutoku K., 2013, The Black Hole-Neutron Star Binary Merger in Full General Relativity: Dependence on Neutron Star Equations of State. Science / Physics / Relativity, Springer Theses

Levkov D. G., Panin A. G., Tkachev I. I., 2018, Phys. Rev. Lett., 121, 151301

Li J., Spitkovsky A., Tchekhovskoy A., 2012, ApJ., 746, 60

Long A. J., Vachaspati T., 2015, Phys. Rev., 91, 103522

Marsh D. J. E., 2016, Phys. Rep., 643, 1

Melrose D. B., Luo Q., 2009, ApJ., 698, 124

Murphy E. J., 2009, ApJ., 706, 482
Nagar A. et al., 2018, Phys. Rev. D, 98, 104052

Nakamura K., 2003, Int. J. Mod. Phys., 18, 4053

Nakar E., Piran T., 2011, Nature, 478, 82

Palenzuela C., Liebling S. L., Neilsen D., Lehner L., Caballero O. L., O’Connor E., Anderson M., 2015, Phys. Rev., 92, 044045

Peccei R. D., Quinn H. R., 1977, Phys. Rev. Lett., 38, 1440

Prentice S. J. et al., 2018, ApJ, 865, L3

Punturo M. et al., 2010, Class. Quantum Gravity, 27, 194002

Raffelt G., Stodolsky L., 1988, Phys. Rev., 37, 1237

Read J. S., Lackey B. D., Owen B. J., Friedman J. L., 2009, Phys. Rev., 79, 124032

Reville B., Kirk J. G., 2010, ApJ., 715, 186

Schive H.-Y., Chiueh T., Broadhurst T., 2014, Nature Phys., 10, 496

Sennett N., Hinderer T., Steinhoff J., Buonanno A., Ossokine S., 2017, Phys. Rev., 96, 024002

Veltmaat J., Niemeyer J. C., Schwabe B., 2018, Phys. Rev., 98, 43509

Widdicombe J. Y., Helfer T., Marsh D. J. E., Lim E. A., 2018, JCAP, 1810 , 005

Wright S. A., Barton E. J., Larkin J. E., Moore A. M., Crampton D., Simard L., 2010, in McLean I. S., Ramsay S. K., Takami H., eds, Proc. SPIE Conf. Ser., Vol. 7735, Ground-based and Airborne Instrumentation for Astronomy III, SPIE, Bellingham, p. 77357P

This paper has been typeset from a $\mathrm{T}_{\mathrm{E}} \mathrm{X} / \mathrm{LT} \mathrm{E} \mathrm{X}$ file prepared by the author. 